Artigo - Seção Estado, Organizações e Sociedade.

\title{
Inteligência Artificial Nos Processos De Seleção De RH
}

\author{
Nome: Gael Santos Cipriano ${ }^{1}$ \\ Universidade Federal de Ouro Preto \\ e-mail: gael.ciprianos@gmail.com
}

Nome: Harrison Bachion Ceribeli ${ }^{2}$

Universidade de São Paulo

Nome: Gustavo Nunes Maciel ${ }^{3}$

Universidade Federal de Lavras

Nome: Alyce Cardoso Campos ${ }^{4}$

Universidade Federal de Lavras

Nome: Rita de Cássia Leal Campos ${ }^{5}$

Centro Federal de Educação MG

\section{RESUMO}

Este estudo teve como objetivo identificar como os softwares desenvolvidos sob a premissa da inteligência artificial podem ser utilizados nos processos de seleção de pessoal e quais benefícios trazem para as organizações. Para isso, realizou-se um estudo de casos múltiplos, contemplando três organizações, duas que ofertam o serviço de seleção a partir de suas plataformas online e uma que desenvolve e comercializa softwares de inteligência artificial. A partir dos dados coletados, constatou-se que os produtos e serviços ofertados pelas empresas que participaram da pesquisa são similares em seus propósitos, tendo como principal finalidade a automatização do processo de seleção, especialmente das etapas de cunho operacional. Além disso, verificou-se a importância da interação humana nas fases iniciais de implantação do programa. As soluções computacionais que foram objeto do presente estudo não se limitam a analisar as informações que constam nos currículos dos candidatos: todas elas buscam traçar um perfil técnico, comportamental e cultural dos candidatos, fornecendo, dessa forma, amplo suporte aos tomadores de decisão.

Palavras chave: seleção de funcionários, aprendizado de máquina, tecnologia aplicada à gestão de pessoas.

\section{ABSTRACT}

This study aimed to identify how software developed under the premise of artificial intelligence can be used in people selection processes and what benefits they bring to organizations. A multiple case study was conducted, contemplating three organizations, two that offer the selection service from their online platforms and one that develops and sells artificial intelligence software. From the collected data, it was found that the products and services offered by the companies that participated in this research are similar in their purposes, having as main objective the automation of the selection process, especially the operational stages. In addition, the importance of human interaction in the early stages of program implementation was verified. The computational solutions that were the object of this study are not limited to the analysis of the information contained in the candidates' curriculum: all of them seek to draw a technical, behavioral and cultural profile of the candidates, thus providing broad support to decision makers.

Keywords: employee selection, machine learning, technology applied to human resource management.

[Submetido em 23-dez-2021 - Aceito em: 22-jan-2022 - Publicado em: 25-fev-2022]

\footnotetext{
'Graduação em Administração (UFOP) [(2020)].

${ }^{2}$ Graduação em Administração (COC) [(2008)], Mestrado em Administração (USP) [(2011)], Doutorado em Administração (USP) [(2013)].

${ }^{3}$ Graduação em Administração (UFOP) [(2017)], Mestrado em Administração (UFLA) [(2020)].

${ }^{4}$ Graduação em Administração (UFJF) [(2017)], Mestrado em Administração (UFLA) [(2020)].

${ }^{5}$ Graduação em Administração (IFMG) [(2016)], Mestrado em Administração (CEFET-MG) [(2019)].
} 


\section{INTRODUÇÃO}

Com a contínua evolução da tecnologia, que deu origem, ao longo do tempo, a mecanismos computacionais cada vez mais autônomos, eficientes e eficazes, alcançou-se o que é considerado o ápice das soluções computacionais na contemporaneidade: a inteligência artificial (Mendonça, Andrade, \& Souza Neto, 2018).

$O$ termo inteligência artificial define o resultado da junção da ciência e da engenharia, que viabilizou a criação de máquinas e programas com a capacidade de desenvolver ações semelhantes à inteligência humana (McCarthy, 2007). Gerenciar a inteligência artificial envolve comunicar, liderar, coordenar e controlar os constantes avanços computacionais para lidar com problemas de tomada de decisão cada vez mais complexos (Berente et al., 2021).

Desde a sua criação, a inteligência artificial evoluiu significativamente e ultrapassou o simplismo de ações em jogos interativos online, alcançando o campo das organizações (Mendonça, Andrade, \& Souza Neto, 2018) e passando a prover suporte em diferentes áreas: desde o atendimento autônomo ao cliente por meio de mecanismos de respostas a perguntas pré-definidas; o auxílio à tomada de decisão gerencial a partir da análise de dados internos e externos do negócio; as previsões mercadológicas (Poisat \& Mey, 2017); a análise do comportamento do consumidor (André et al, 2018); até a automatização dos processos de gestão de pessoas (Strohmeier \& Piazza, 2015).

Nos últimos anos, é possível observar o uso da inteligência artificial nos processos de seleção de candidatos, principalmente nas organizações em que há a valorização da gestão de pessoas em uma perspectiva estratégica (Armstrong, Landers, \& Collmus, 2016) e, por conseguinte, a preocupação com questões como atração e retenção de talentos, alinhamento de valores e expectativas entre empregados e empregador, e aspectos psicológicos e comportamentais dos funcionários (Jeske \& Shultz, 2016).

Diante da escassez de estudos relacionados a esse tema, conforme observado no levantamento bibliográfico realizado em bases consolidadas, como Spell, Scopus, Scielo e Redalyc, levantou-se o seguinte questionamento: como os softwares de inteligência artificial podem ser aplicados nos processos de seleção de novos funcionários? Com o intuito de responder a este problema de pesquisa, definiu-se como objetivo deste estudo identificar como os softwares desenvolvidos sob a premissa da inteligência artificial podem ser utilizados nos processos de seleção de pessoal e quais benefícios trazem para as organizações.

A justificativa para a presente pesquisa reside em diferentes aspectos. Primeiramente, no campo teórico, fundamenta-se na carência de estudos abordando a temática aqui explorada, tanto em âmbito nacional quanto internacional, apesar do crescente interesse das empresas em relação ao uso de novas tecnologias em seus processos de gestão. Já na esfera gerencial, visualiza-se a possibilidade de fomentar o debate a respeito da automatização dos processos de gestão de pessoas, de modo que os profissionais que atuam na área possam dedicar-se às atividades mais estratégicas para os negócios.

\section{SELEÇÃO DE PESSOAL NAS ORGANIZAÇÕES}

Os estudos que tratam da seleção de recursos humanos no campo das Ciências 
Administrativas não são recentes. Segundo Dunnette e Borman (1979), o processo de seleção de pessoal começou a ser estudado a partir de 1917, com a observação dos métodos empregados para escolha de oficiais e combatentes americanos que seriam designados para a guerra contra a Alemanha. A temática, todavia, evoluiu bastante, apesar de ainda haver espaço para novas pesquisas abordando métodos, sistemas e ferramentas que sejam capazes de tornar o processo mais efetivo.

Tradicionalmente, conceituou-se a seleção de pessoal como uma combinação de métodos e ações direcionadas para subsidiar a escolha dos novos funcionários de uma organização (Huber, Neale, \& Northcraft, 1987), ou como o processo que visa destinar a pessoa certa para o trabalho certo (De Feis, 1987). Entretanto, o conceito evoluiu recentemente e passou a ser delimitado como o compilado de ações de cunho estratégico de uma organização voltadas para selecionar indivíduos cujas características e competências, técnicas e pessoais, sejam capazes de contribuir com o sucesso corporativo (Weinstein, 2012). Sob essa perspectiva, a seleção deixou de ser vista como um sistema meramente classificatório e eliminatório de candidatos, adquirindo importância estratégica para tornar a qualidade da seleção uma vantagem competitiva para a organização (Azar et al., 2013; Hamza et al., 2021).

Tornando seus processos seletivos mais eficazes, a organização pode ampliar sua capacidade de alcançar os objetivos traçados, à medida que passa a contar com melhores profissionais e se torna mais competitiva perante seus concorrentes (Redondo, 2014). Além disso, um processo seletivo com enfoque estratégico possibilita o fortalecimento da cultura organizacional, dando preferência a candidatos que possuem valores pessoais alinhados aos do negócio (Brannan \& Hawkins, 2007).

Complementarmente, observa-se também uma estreita relação entre a seleção de pessoal e a manutenção ou mesmo expansão do potencial produtivo das organizações (Ahmad \& Schroeder, 2002), visto que o referido processo fornece o subsídio necessário para o aprimoramento das competências organizacionais ao incorporar novas competências humanas ao negócio (Alonso et al., 2015).

Logo, ultrapassando a concepção simplista de que a seleção de pessoal tem por objetivo apenas identificar o profissional mais capacitado para desempenhar uma função, verifica-se que este processo assume uma posição essencial dentro do conjunto de práticas que tem por intuito o desenvolvimento, a consolidação e o crescimento de qualquer organização (Contreras, Almaguer, \& Tovar, 2015; Kleinmann et al., 2011).Todavia, além das vantagens estratégicas, salienta-se o impacto dos processos de seleção sobre os custos organizacionais, dado que podem aumentar ou diminuir os dispêndios financeiros da organização relacionados à contratação, desligamento e capacitação de funcionários (Kleinmann et al. 2011).

Observa-se, portanto, que a seleção de pessoal não se trata apenas de contratar o profissional adequado para ocupar determinado cargo; é também um sistema estratégico e importante mecanismo para consolidação e crescimento do negócio (Bolander \& Sandberg, 2013). E, ante a relevância do referido processo, é natural que gestores e profissionais da área de gestão de pessoas dediquem-se à busca de novas soluções, tendo como intuito torná-lo mais eficaz e eficiente (Lievens, Van Dan, \& Anderson, 2002).

Perpassando pelos métodos seletivos empregados pelas organizações ao longo do tempo, é possível observar a diversidade de alternativas utilizadas e como evoluíram, acompanhando o desenvolvimento tecnológico alcançado pela sociedade (Mariani, Chiesa, \& Gill, 2017). No final da década de 1980, por exemplo, percebia-se a necessidade de 
modificação e aprimoramento dos métodos de seleção existentes, para que permanecessem adequados diante das constantes transformações do mercado. Tal constatação levou Van Dyke e Strick (1988) a recomendarem que a verificação de referências com antigos empregadores fosse substituída ou pelo menos complementada pela aplicação de testes pré-seletivos.

Mais adiante, em meados da década de 1990, Shackleton e Newell (1994) retrataram os métodos que passaram a ser recorrentemente utilizados naquele período, como a contratação de centros de avaliação terceirizados e o uso de testes psicológicos, de dados biográficos e da grafologia. A opção por centros de avaliação perdurou por mais de 10 anos, conforme se verifica em Robertson e Smith (2001), devido à praticidade e ausência de profissionais da área da Psicologia que estivessem inseridos no meio empresarial. Por sua vez, a aplicação sistemática de testes psicológicos foi apontada por Fisher e Boyle (1997) como importante ferramenta de suporte ao processo de seleção de empregados nas organizações.

Posteriormente, com o intuito de se obter maior suporte para a tomada de decisão nos processos seletivos das organizações, passaram a ser utilizados jogos interativos, que ficaram conhecidos como role-play (ou dinâmicas) e simulavam, de forma lúdica, o ambiente laboral e as funções a serem desempenhadas (Brannan \& Hawkins, 2007), além de games que permitissem a avaliação de fatores psicológicos e comportamentais dos candidatos (Armstrong, Landers, \& Collmus, 2016), e abordagens focadas nas competências individuais (Alonso et al., 2015).

Além disso, os profissionais responsáveis pela seleção de pessoal nas organizações passaram a fazer uso das informações disponíveis nas redes sociais, cruzando-as com as que constavam nos currículos dos indivíduos, com o intuito de traçar perfis comportamentais (Jeske \& Shultz, 2016). As empresas podem se beneficiar das vantagens das mídias sociais em termos de redução de tempo e custo, porém é preciso atentar para questões de ética e segurança. As informações disponíveis em redes sociais trazem uma série de questões jurídicas. Além disso, permitem facilmente que os preconceitos individuais afetem as decisões de contratação e triagem (Emanuela, 2018).

Novas ferramentas foram desenvolvidas para explorar de maneira mais apropriada os dados dos candidatos que poderiam ser levantados (Berkelaar, 2017; Jeske \& Shultz, 2016; Machado, 2016; Roth et al., 2016). Além disso, foram criadas promissoras técnicas para captação, organização e análise de dados disponibilizados em rede, especialmente nas mídias sociais, cuja aplicação ficou conhecida como cybervetting, tornando os processos de seleção de pessoal mais efetivos (Azar et al., 2013). De maneira complementar, incorporou-se aos processos seletivos o uso de big data, com o objetivo de atender a demanda por novas ferramentas e sistemas de tecnologia da informação (Derous \& Fruyt, 2016), mineração de dados (Azar et al., 2013), algoritmos de triagem (Strohmeier \& Piazza, 2015) e inteligência artificial (Buzko et al., 2015). Porém, é necessária uma reflexão crítica acerca da utilização de informações das redes sociais dos candidatos, podendo pesar implicações éticas. Esta prática pode consolidar mecanismos de controle panópticos, gerando desconfiança e insegurança que podem refletir na própria organização para a qual a seleção está sendo conduzida quanto na sociedade como um todo.

Ainda pouco usual, a inteligência artificial, que se refere a qualquer dispositivo computacional que simula a ação e racionalidade do pensamento humano (Strohmeier \& Piazza, 2015), trouxe para o campo das organizações novas possibilidades para tornar o 
processo decisório mais efetivo (Mendonça, Andrade, \& Souza Neto, 2018). E, como ramificações desse campo, surgiram as redes neurais e o aprendizado de máquina, ambos mecanismos desenvolvidos visando oportunizar e desenvolver nas máquinas a capacidade de aprender, assim como ocorre com os humanos (Huang, 2014).

Graças aos sistemas de inteligência artificial, a tecnologia passou a permitir a assimilação e consolidação do conhecimento a partir de informações concretas disponíveis em um banco de dados (Hassabis, 2017; Soete, Lievens, \& Druart, 2013). Por isso, sua aplicabilidade no meio empresarial mostra-se cada vez mais promissora (Mendonça, Andrade, \& Souza Neto, 2018) e se expande diante dos desafios econômicos, sociais, mercadológicos, globais e estratégicos que as organizações enfrentam na contemporaneidade para se manterem competitivas e lograrem êxito (Alonso et al., 2015).

O uso da inteligência artificial nos processos de seleção de pessoal pode ser apontado como um marco da evolução que tem sido alcançada nessa área, fornecendo cada vez mais subsídios para que as organizações encontrem o "candidato ótimo" (Strohmeier \& Piazza, 2015) por meio de um mapeamento preliminar do perfil psicocomportamental dos indivíduos alinhado à cultura organizacional e associado a uma análise das competências técnicas e interpessoais necessárias para o negócio (Karatop, Kubat, \& Uygun 2015).

Diante de temas como gênero, orientação sexual, raça, equidade e igualdade de direitos, que estão cada vez mais presentes nas discussões no meio empresarial e persistem como um desafio para os gestores, o emprego da inteligência artificial nos diferentes processos de gestão de pessoas, entre eles a seleção, pode trazer ganhos significativos tanto para as organizações quanto para os profissionais (Buzko et al., 2015). De acordo com Atanazio et al. (2021), um ambiente empresarial saudável é aquele que está aberto a uma constante transformação cultural, aberto a novos olhares e tecnologias. As empresas existem com o intuito de satisfazer a necessidade humana e as pessoas mudam seus costumes e modo de agir perante a sociedade. Assim, as organizações também devem ser passíveis de evolução.

O uso de inteligência artificial vem crescendo em alguns países. No caso do Brasil, desde 2015, o número de contratações de desenvolvedores de inteligência artificial cresceu mais que o dobro (Valente, 2020). Casos de sucesso com a utilização no recrutamento são, por exemplo, nas empresas brasileiras Time-Now e Cotesa, além da Embraer que atua em 60 países (Alves, 2021). Porém, mesmo crescendo na utilização, o Brasil ainda está fora da lista de países com grandes conglomerados digitais, buscando aumentar a inovação em inteligência artificial. Nos Estados Unidos, mais de US\$30 bilhões foram investidos em iniciativas no ramo entre 2018 e 2019, na China US $\$ 25$ bilhões e no Reino Unido US $\$ 5$ bilhões. Segundo relatório da Brasscom, a perspectiva de investimento em inteligência artificial é de $R \$ 2,5$ bilhões entre 2018 e 2022 (Agência Brasil, 2020).

Reis e Graminho (2019) e Kirchner (2020), em suas pesquisas com a multinacional Amazon e a utilização de inteligência artificial para a contratação de funcionários, apontam como atuar com a proteção de dados pode funcionar como um limite ao uso abusivo de poder e a não violação dos direitos fundamentais, tendo um cuidado cada vez maior por parte dos programadores. Aguiar, Raupp e Macedo (2019), em seu estudo realizado com uma empresa brasileira, afirmam que inúmeros são os benefícios para o processo de recrutamento e seleção e deve ser disseminado para as demais áreas do RH. 
O estudo realizado em Cuba por Torres-López et al. (2014) mostra como os problemas associados aos fatores humanos são os que constituem o de maior incidência nos resultados dos projetos. Assim, além de colaborar no momento de seleção, pode continuar auxiliando no momento de gestão dos recursos humanos da empresa.

Alguns autores defendem que, por se tratar de uma ferramenta desenvolvida sob a perspectiva de uma ciência exata, mitiga-se a subjetividade normalmente existente nos processos seletivos (Alves et al., 2015), considerando que se reduz a influência dos preconceitos e padrões inerentes aos tomadores de decisão (Poisat \& Mey, 2017; Atanazio et al., 2021). Assim, um processo de contratação às cegas possibilita a abertura de espaço nas organizações no quesito diversidade e inclusão (Atanazio et al., 2021). Em sistemas baseados na inteligência artificial, a probabilidade de subjetividade real é muito pequena. Porém, deve ser considerado, pois softwares precisam ser alimentados por indivíduos que são subjetivos. Assim, não é possível neutralizar por completo o processo.

Diversos pontos positivos na utilização de inteligência artificial podem ser citados, como a capacidade de comparar de forma rápida os fatos e identificar as relações devido à alta velocidade de processamento das informações (Evseeva et al., 2021). A inteligência artificial tem sido utilizada em grande escala por empresas especializadas a fim de alcançar eficácia e custo-benefício. Todavia, assim como toda tecnologia, a adoção da inteligência artificial nos processos de recrutamento e seleção também apresenta riscos, uma vez que pode causar desconfiança tanto entre os recrutadores como entre os candidatos (Ore \& Sposato, 2021). Sendo assim, ao mesmo tempo em que aproveitam as vantagens das ferramentas baseadas em inteligência artificial, as empresas devem se preocupar com a relação interpessoal, tranquilizando os candidatos em relação às máquinas e tornando o processo mais amigável e confortável (Jha, Jha \& Gupta, 2020).

\section{ASPECTOS METODOLÓGICOS}

A presente pesquisa possui caráter descritivo, dado que estudos dessa natureza buscam descrever precisamente os fatos e fenômenos de determinada realidade (Triviños, 2008), que neste caso é a inteligência artificial e como ela pode ser empregada nos processos de seleção de pessoal nas organizações. Optou-se por uma abordagem qualitativa, à medida que se objetivou compreender de maneira aprofundada a percepção dos sujeitos envolvidos com o objeto examinado (Zanella, 2009).

O método escolhido para investigar o objeto de pesquisa delimitado foi o estudo de casos múltiplos, apontado por Gil (2010) como um dos meios de analisar casos diversos acerca dos quais se almeja aprofundar o conhecimento e que possibilita, ao considerar os respectivos contextos, levantar um arcabouço de variáveis potencialmente relevantes. Essa lógica difere-se daquela que direciona o estudo de caso único, em que se parte de uma perspectiva bem definida ou considera casos extremamente raros. Para Yin (2015), esse método aumenta a validade externa da pesquisa, pois produz resultados mais convincentes e permite maiores generalizações.

O estudo de caso é um método amplamente empregado nas pesquisas da Administração, pois busca responder questões do tipo "como" e "por que" em cenários nos quais as variáveis relacionadas estão intrínsecas a um contexto verossímil e não hipotético, não sendo possível, portanto, estudar o fenômeno de maneira descontextualizada (Yin, 2015).

Para o desenvolvimento do estudo foram escolhidas três organizações: duas que 
ofertam o serviço de seleção a partir de suas plataformas online e uma que desenvolve e comercializa softwares de inteligência artificial, as quais foram selecionadas a partir de um levantamento em meio virtual, utilizando-se como critério de escolha a maior disponibilização de informações nas páginas institucionais. Para garantia do anonimato das organizações escolhidas, estas foram denominadas como A, B e C.

A empresa A foi fundada no ano de 2015 e possui atualmente 35 colaboradores. Está localizada na cidade de São Paulo e oferta serviços de recrutamento, com anúncio das vagas, e seleção, perpassando por etapas como análise curricular, testes e dinâmicas de grupo, com dados analisados pelo software de inteligência artificial.

A organização definida como B tem atuação global e iniciou suas atividades no Brasil no ano de 1917. Tem mais de 500 funcionários atuantes no país, com sede na cidade de São Paulo e filiais em Brasília, Minas Gerais, Rio de Janeiro e Rio Grande do Sul. Os principais produtos e serviços estão vinculados ao desenvolvimento de tecnologias computacionais, softwares, hardwares, computadores, segurança, business solutions e correlatos.

A última organização contatada, aqui denominada como $\mathrm{C}$, foi fundada no ano de 1999. Está localizada na cidade de São Paulo, possui 35 funcionários e tem como principal serviço à oferta de soluções tecnológicas para recrutamento e seleção de pessoas.

Com base nos estudos preliminares sobre a seleção de pessoas, seus processos e evolução, e nas informações observadas nos sites das empresas, elaborou-se o roteiro de entrevista, contendo perguntas não diretivas, abertas, utilizando questões cujas respostas ultrapassassem o simplismo do sim ou não, buscando maior entendimento sobre o fenômeno aqui tratado, referente ao uso da inteligência artificial nos processos de seleção de pessoal nas organizações por meio das soluções computacionais desenvolvidas e comercializadas pelas empresas que participaram da pesquisa.

Como método de coleta de dados, optou-se pela entrevista em profundidade, uma vez que esta, como posto por Oliveira, Martins e Vasconcelos (2012), seria a ferramenta mais adequada diante de um contexto em que se há pouco conhecimento, sendo necessário um maior aprofundamento, que só se concretiza com a contribuição do entrevistado e sua percepção diante do fato ou objeto observado.

Para tal, foi solicitado, via e-mail e por contato telefônico, agendamento com um colaborador da organização para participação no estudo. Os entrevistados foram identificados conforme as organizações que atuam. O entrevistado A faz parte do corpo de fundadores e ocupa o cargo de relações públicas na empresa, sendo responsável pela imagem institucional junto a agentes externos, mídias e afins. O entrevistado B é engenheiro de software, atua no setor de aprimoramento e desenvolvimento de ferramentas para o setor de negócios, e trabalha na empresa desde 2010. O colaborador C ocupa o cargo de Customer Success e está na instituição desde 2016. As entrevistas foram gravadas e posteriormente transcritas para então serem analisadas.

Utilizou-se como técnica para exploração das respostas a análise de conteúdo, podendo esta ser definida como o coletivo de métodos e ferramentas que visam analisar informações advindas de diferentes fontes (Silva \& Fossá, 2015). Neste estudo, as fontes foram verbais, adquiridas por meio de entrevista em profundidade com três indivíduos não correlacionados.

A análise de conteúdo compila as possibilidades de verificação das informações 
coletadas, podendo ser executada a partir de unidades referenciais diversas, tais como unidades sintáticas, unidades proposicionais e unidades temáticas, sendo esta última escolhida para o desenvolvimento desse estudo, uma vez que possibilita a identificação de padrões por meio de recortes textuais apurados na transcrição das entrevistas (Zanella, 2006).

Observado o proposto por Bardin (2011), seguiram-se as seguintes etapas para verificação dos dados: pré-análise, exploração do material e interpretação. Cabe esclarecer que a primeira etapa corresponde à organização e sistematização do material coletado, seguida pela exploração dos dados de acordo com unidades temáticas, que, neste estudo, originaram-se da divisão das perguntas do roteiro de entrevista de acordo com o tema central abordado; por fim, tem-se a interpretação, realizando a comparação entre o que foi observado e o referencial teórico consultado (Oliveira, Martins, \& Vasconcelos, 2012).

\section{RESULTADOS}

Por meio das entrevistas em profundidade realizadas, foram coletadas informações que permitiram ampliar o conhecimento relacionado ao funcionamento, operacionalização e aplicação dos softwares de inteligência artificial desenvolvidos pelas três empresas que colaboraram com este estudo. No que tange à caracterização dos softwares, o entrevistado B aponta que, de uma forma simplista, a estruturação do software se dá pelo alinhamento entre um banco de dados contendo informações sobre uma demanda específica, neste caso, um banco de currículos, e sequencialmente pela alimentação da tecnologia de inteligência artificial com essas informações de forma repetitiva. A cada currículo selecionado para a próxima etapa, o software realiza uma leitura das informações contidas no documento e, caso ela se repita, ele identifica que aquelas características atendem aos requisitos da vaga.

De forma similar ocorre o funcionamento do software da empresa A. O entrevistado relata que a operacionalização se dá através da alimentação do banco de dados em uma página criada para o contratante, na qual é realizado um levantamento do perfil do candidato desejado, dentro das exigências indicadas pelo cliente. Em um primeiro momento, o software recebe comandos que irão condicioná-lo a fazer a leitura dos requisitos da vaga e, posteriormente, ele adquire um comportamento automático. $\mathrm{O}$ entrevistado A acrescentou que:

"Para formação dos indicadores de condicionamento do software, fazemos mapeamento do perfil técnico e comportamental dos funcionários que o contratante já possui, através de um questionário e expondo eles ao mesmo processo a que os demais candidatos serão condicionados. A partir deste, o software de inteligência artificial desenvolve a habilidade de identificar na triagem curricular, testes de conhecimento, perfil e games, o candidato que melhor se adequa às necessidades técnicas, ao mesmo tempo em que avalia o 'fit cultural' entre as partes".

Mecanismo semelhante a esse é observado no software da empresa $\mathrm{C}$, em que um banco de dados, que compila as informações dos candidatos, é integrado a um sistema de inteligência artificial responsável pela seleção dos melhores currículos conforme 
demandas dos contratantes, considerando conhecimentos específicos e técnicos necessários para o desenvolvimento das atividades, além das habilidades interpessoais, alinhamento cultural e de valores, que são mapeados por meio de testes e games integrados à ferramenta.

Fica evidente na fala dos entrevistados a necessidade da interação humana com os softwares, principalmente nas fases iniciais de implantação, que são caracterizadas como etapa de aprendizado das soluções de (IA). Um dos trechos da entrevista realizada com o representante da empresa B salienta essa questão:

"O software precisa de interação humana, inicialmente no lançamento de informações para direcioná-lo, posteriormente para a seleção de informações e de dados que são necessários para que ele crie um processo de aprendizagem autônomo para tomar decisões de maneira autônoma caso seja exposto àquela mesma situação ou outra semelhante".

Para avaliação das habilidades interpessoais e mapeamento de valores e alinhamento cultural dos candidatos, o software da empresa C utiliza games para condução de dinâmicas online, que possibilitam a análise de comportamento em grupo, liderança, trabalho em equipe, proatividade, tomada de decisão, além de testes psicológicos, como o teste DISC e o Questionário de Avaliação Psicológica.

Já o software da empresa A utiliza um teste criado pela própria organização para mapeamento comportamental dos candidatos, que consiste em um conjunto de afirmativas vinculadas a uma escala de cinco pontos que mede o grau de afinidade do respondente com cada item apresentado. As afirmativas abordam situações extremas, como trabalhar 12 horas consecutivas; desejos, como ter um carro importado ou fazer uma viagem pelo mundo; e preferências individuais, como remuneração atrelada à meritocracia ou flexibilidade no trabalho e mais tempo para família.

A solução desenvolvida pela empresa $\mathrm{C}$ utiliza as informações disponíveis nas redes sociais, prática que está se tornando cada vez mais recorrente nos processos de seleção (Jeske \& Schultz, 2016). Conforme relatou o entrevistado C: "No nosso sistema, o candidato aponta suas preferências e registra endereços de suas mídias sociais; o sistema, sequencialmente, armazena essas informações, que são tratadas através da parte do software que identificamos como inteligência cognitiva".

De modo semelhante, a empresa B adota um software complementar ao sistema de inteligência artificial, que oportuniza a análise de dados disponíveis na rede (redes sociais e aplicativos de áudio e vídeo). Um exemplo foi exposto pelo representante da organização:

"De acordo com as postagens, comentários, grupos e outros fatores e movimentos em rede, o software de inteligência artificial, conjuntamente ao dispositivo complementar, consegue identificar o perfil comportamental do indivíduo; basta que se tenha o endereço de uma página de mídia social ou sites correlatos. Todas essas informações visam formar, para o cliente, um banco com o perfil mais adequado técnica e culturalmente para ocupar determinado cargo". 
Porém, uma reflexão crítica sobre a utilização de informações das mídias sociais dos candidatos de ser realizada, por preocupação ética. Esta prática pode fortalecer mecanismos de controle que poderão não somente prejudicar os candidatos, como também manchar a imagem da organização que está realizando o processo.

Em relação às vantagens do uso da inteligência artificial nos processos de seleção de pessoal nas organizações, os entrevistados apresentaram as mesmas percepções, levantando aspectos como redução do tempo gasto no processo de análise curricular e na aplicação e avaliação dos resultados de testes psicológicos e provas de conhecimentos, além de redução do viés na escolha dos candidatos em decorrência de preconceitos, uma vez que os softwares, caso sejam devidamente alimentados, não consideram no processo de tomada de decisão questões como etnia, orientação sexual, gênero e outros.

Comparando as ferramentas de (IA) ao uso de sites de vagas e de redes profissionais, como o Linkedin, o entrevistado B explicou que:

"Esse tipo de plataforma faz o cruzamento entre oferta e demanda, e às vezes sinaliza o melhor perfil a partir de uma base algorítmica limitada. Em contrapartida, quando se utiliza inteligência artificial, o processo é mais amplo, pois não há um algoritmo limitante e sim um algoritmo que impulsiona a aprendizagem do software e não está focado em um grupo específico de informações".

$\mathrm{Na}$ mesma linha, o entrevistado A relatou que "tais aplicativos focam em requisitos técnicos e, atualmente, há uma mudança na demanda de contratação, que engloba o alinhamento cultural e a avaliação de quesitos comportamentais", enquanto o entrevistado $\mathrm{C}$ assinalou que:

"A principal diferença está na potencialidade dos softwares de inteligência artificial de ultrapassarem o simplismo da avaliação curricular, que ocorre nas plataformas convencionais, pois os resultados entregues abrangem capacidade técnica, experiências anteriores e habilidades interpessoais".

Ainda que os softwares se proponham a entregar todos os benefícios e vantagens ora mencionados, questionaram-se os entrevistados a respeito de possíveis limitações dos sistemas de (IA). Segundo o entrevistado B, "o valor agregado encarece o serviço e o torna inacessível para algumas organizações, principalmente as de médio e pequeno porte", sendo essa percepção compartilhada pelo entrevistado A. Já para o entrevistado C, além do alto valor de investimento necessário, tem-se que se considerar ainda que a alimentação inadequada do software pode perpetuar comportamentos, como diferenciação de gênero, faixa etária, instituição de ensino, entre outros.

Analisando os dados levantados junto aos representantes das organizações que se dispuseram a colaborar com a presente pesquisa, constatou-se que as soluções de inteligência artificial visam tornar o processo de seleção mais autônomo, principalmente no que diz respeito às etapas de caráter mais operacional, tais como triagem curricular e aplicação de testes psicológicos e provas de conhecimento, à medida que possibilitam uma análise do perfil técnico-comportamental dos candidatos e a escolha daquele que melhor se adéqua às necessidades não apenas técnicas, mas também culturais da empresa 
contratante (Karatop, Kubat, \& Uygun, 2015).

Percebe-se, portanto, que tais soluções podem ser apontadas como um marco evolutivo dos processos de seleção de pessoal, considerando que favorecem a identificação dos profissionais que potencialmente irão se ajustar melhor e agregar mais competências à organização (Strohmeier \& Piazza, 2015).

Apesar de a inteligência artificial ser vista como um campo promissor, as reais potencialidades e limitações do aprendizado autônomo da máquina no que diz respeito aos processos de seleção de pessoal ainda carecem de investigação, pois, conforme assinala (Mendonça, Andrade, \& Souza Neto, 2018), trata-se, por ora, de uma ferramenta pouco usual, cuja efetiva contribuição para os processos decisórios e estratégicos das organizações ainda se encontra em território desconhecido.

\section{CONSIDERAÇÕES FINAIS}

Com o desenvolvimento da inteligência artificial e aprendizagem das máquinas, chegaram ao campo dos negócios soluções que prometem otimizar a tomada de decisão e os diferentes processos de gestão, incluindo o processo de seleção de pessoal. O uso dessas ferramentas mostra-se promissor, mas, apesar disso, foi, até o presente momento, pouco abordado na literatura, principalmente no âmbito da Ciência Administrativa.

Diante desse contexto, definiu-se como objetivo de pesquisa identificar como os softwares desenvolvidos sob a premissa da inteligência artificial podem ser utilizados nos processos de seleção de pessoal e quais benefícios trazem para as organizações. A partir das entrevistas realizadas, constatou-se que os produtos e serviços ofertados pelas empresas que participaram da pesquisa são similares em seus propósitos, tendo como principal finalidade a automatização do processo de seleção, especialmente das etapas de cunho operacional, como gerenciamento do banco de currículos; triagem de currículos; aplicação de testes psicológicos e de conhecimentos; mapeamento do perfil comportamental e cultural dos candidatos; e cruzamento de dados com informações disponíveis em rede. Além disso, verificou-se a importância da interação humana nas fases iniciais de implantação do programa, para que ele consiga identificar padrões a serem replicados em futuros processos seletivos.

Finalmente, observou-se também que as soluções computacionais que foram objeto do presente estudo não se limitam a analisar as informações que constam nos currículos dos candidatos, ou seja, não se resumem a mecanismos de filtro. Ao contrário, todas elas buscam traçar um perfil técnico, comportamental e cultural dos candidatos, fornecendo, dessa forma, amplo suporte aos tomadores de decisão.

A contribuição desta pesquisa para o campo gerencial encontra-se na possibilidade de chamar a atenção dos gestores e estimulá-los a refletir a respeito dos benefícios potenciais de utilizar softwares de inteligência artificial nos processos de seleção de pessoal nas organizações. Em outra perspectiva, nota-se uma contribuição também para a Ciência Administrativa, fornecendo novos elementos à discussão acerca do uso de tecnologias computacionais para dar suporte aos processos de gestão de pessoas, ao mesmo tempo em que fomenta novas investigações nesta área, considerando que se trata de uma linha de pesquisa promissora.

Para estudos futuros, sugere-se que sejam analisadas as percepções dos profissionais que atuam na área de gestão de pessoas em organizações que fazem uso de 
soluções de inteligência artificial nos processos de seleção, com o intuito de levantar os reais benefícios que podem ser atribuídos a essa tecnologia. Complementarmente, propõe-se ainda que sejam examinados, nestas mesmas organizações, os possíveis focos de resistência e as dificuldades para implantação desses softwares. Além disso, outra sugestão é a realização de um estudo sobre a percepção dos candidatos submetidos aos processos seletivos orientados pelas soluções de inteligência artificial.

\section{REFERÊNCIAS}

Agência Brasil. Brasil está atrasado na corrida por inteligência artificial. Correio Braziliense. https://www.correiobraziliense.com.br/tecnologia/2020/09/4872467-brasilesta-atrasado-na-corrida-por-inteligencia-artificial.html

Aguiar, F. F., Raupp, D. S., \& Macedo, M. (2019). A transformação digital no setor de recursos humanos: um estudo de caso sobre o uso da tecnologia no processo de recrutamento e seleção. Ciki, 1-15.

Ahmad, S., \& Schroeder, R. G. (2002) The importance of recruitment and selection process for sustainability of total quality management. International Journal of Quality \& Reliability Management, 19(5), 540-550.

Alonso, M. F., Padilla, M. V., Bermúdez, G. M. D., Simón, N. D., \& Hernández, H. S. (2015). Perfil por competencias laborales y modelo de selección de personal para el cargo Técnico A en Gestión de Recursos Humanos. Wimblu, 10(2), 19-37.

Alves, R., Silva, F., Mota, D., Mysmar, D., \& Alves, S. (2015). Seleção de pessoas por meio de algoritmos genéticos. Revista de Administração da Universidade Federal de Santa Maria, 10(2), 307-317.

Alves, V. Conheça 3 histórias de sucesso do uso de I.A no recrutamento. Gupy. https://www.gupy.io/blog/historias-de-sucesso-i-a

André, Q., Carmon, Z., Wertenbroch, K., Crum, A., Frank, D., Goldstein, W., Huber, J., Boven, L. V., Weber, B., \& Yang, H. (2018). Consumer choice and autonomy in the age of artificial intelligence and big data. Customer Needs and Solutions, 5(1-2), 28-37.

Armstrong, M. B., Landers, R. N., \& Collmus, A. B. (2016). Gamifying recruitment, selection, training, and performance management: Game-thinking in human resource management. In Emerging research and trends in gamification (pp. 140-165). IGI Global.

Atanazio, A,. Silva, L. R. O., Formigoni, A., Novais, R. A. B. A inteligência artificial transformando o RH do futuro: um estudo de caso sobre a tecnologia e a diversidade no mercado de trabalho. Refas, 7(4), 1-16, 2021.

Azar, A., Rajaeian, A., Sebt, M. V., \& Ahmadi, P. (2013). A model for personnel selection with a data mining approach: A case study in a commercial bank. SA Journal of Human Resource Management, 11(1), 1-10.

Bardin, L. (2011). Análise de conteúdo. São Paulo: Edições 70.

Berente, N., Gu, B., Recker, J., \& Santhanam, R. (2021). Managing Artificial Intelligence. MIS Quarterly, 45(3).

Berkelaar, B. L. (2017). Different ways new information technologies influence conventional organizational practices and employment relationships: The case of cybervetting for personnel selection. Human Relations, 70(9), 1115-1140. 
Bolander, P., \& Sandberg, J. (2013). How employee selection decisions are made in practice. Organization Studies, 34(3), 285-311.

Brannan, M. J., \& Hawkins, B. (2007). London calling: selection as pre-emptive strategy for cultural control. Employee Relations, 29(2), 178-191.

Buzko, I., Dyachenko, Y., Petrova, M., Nenkov, N., Tuleninova, D., \& Koeva, K. (2016). Artificial Intelligence technologies in human resource development. Computer Modelling and New Technologies, 20(2), 26-29.

Contreras, C. A. R., Almaguer, K. P. J., \& Tovar, Y. S. (2015). Perceptions of the impact of training, compensation and recruitment on project effectiveness. AD-minister, 27, 81104.

Derous, E., \& Fruyt, F. D. (2016). Developments in recruitment and selection research. International Journal of Selection and Assessment, 24(1), 1-3.

Dunnette, M. D., \& Borman, W. C. (1979). Personnel selection and classification systems. Annual review of psychology, 30(1), 477-525.

De Feis, G. L. (1987). People: an invaluable resource. Journal of Management in Engineering, 3(2), 155-162.

Emanuela, T. A. (2018). The impact of social media on recruitment. Ovidius" University Annals, Economic Sciences Series, 18(2), 537-541.

Evseeva, S., Evseeva, O., Burmistrov, A., \& Siniavina, M. (2021). Application of artificial intelligence in human resource management in the agricultural sector. In E3S Web of Conferences (Vol. 258). EDP Sciences.

Fisher, C. D., \& Boyle, G. J. (1997). Personality and employee selection: Credibility regained. Asia Pacific Journal of Human Resources, 35(2), 26-40.

Gil, A. C. (2010). Como elaborar projetos de pesquisa. São Paulo: Atlas, 5.

Hamza, P. A., Othman, B. J., Gardi, B., Sorguli, S., Aziz, H. M., Ahmed, S. A., ... \& Anwar, G. (2021). Recruitment and Selection: The Relationship between Recruitment and Selection with Organizational Performance. International Journal of Engineering, Business and Management, 5(3), 1-13.

Hassabis, D. (2017). Artificial Intelligence: Chess match of the century. Nature, 544(7651), 413-414.

Huang, G. B. (2014). An insight into extreme learning machines: random neurons, random features and kernels. Cognitive Computation, 6(3), 376-390.

Huber, V. L., Neale M. A., \& Northcraft G. B. (1987). Decision bias and personnel selection strategies. Organizational Behavior and Human Decision Processes, 40(1), 136147.

Jha, S. K., Jha, S., \& Gupta, M. K. (2020, March). Leveraging Artificial Intelligence for Effective Recruitment and Selection Processes. In International Conference on Communication, Computing and Electronics Systems (pp. 287-293). Springer, Singapore.

Jeske, D., \& Shultz, K. S. (2016). Using social media content for screening in recruitment and selection: pros and cons. Work, employment and society, 30(3), 535-546.

Karatop, B., Kubat, C., \& Uygun, Ö. (2015). Talent management in manufacturing system using fuzzy logic approach. Computers \& Industrial Engineering, 86, 127-136. 
Estudos DE ADMINISTRAÇÃo E SociedADE V.6, N.2 (2021) p.8 - p.22

Kirchner, I. L. (2020). O uso da inteligência artificial sob a ótica dos direitos fundamentais: análise do caso Amazon [Trabalho de Conclusão de Curso, Universidade Federal do Rio Grande do Sul]. Lume Repositório Digital. https://www.lume.ufrgs.br/handle/10183/221925

Kleinmann, M., Ingold, P. V., Lievens, F., Jansen, A., Melchers, K. G., \& König, C. J. (2011). A different look at why selection procedures work: The role of candidates' ability to identify criteria. Organizational Psychology Review, 1(2), 128-146.

Lievens, F., Van Dam, K., \& Anderson, N. (2002). Recent trends and challenges in personnel selection. Personnel review, 31(5), 580-601.

Machado, L. M. O. (2016). A influência da informação publicada nas "redes sociais" no processo de seleção e recrutamento: um estudo exploratório na literatura. AtoZ: novas práticas em informação e conhecimento, 5(1), 10-20.

Mariani, M. G., Chiesa, R., \& Gill, H. (2017). Self-efficacy and justice perceptions in personnel selection: A moderated mediation model. International Journal of Selection and Assessment, 25(2), 203-211.

Mendonça, C. M., Andrade, A. M. V., \& Souza Neto, M. V. (2018). Uso da IoT, Big Data e inteligência artificial nas capacidades dinâmicas. Revista Pensamento Contemporâneo em Administração, 12(1), 131-151.

McCarthy, J. (2007). What is Artificial Intelligence? Computer Science Department, Stanford University.

Oliveira, V. M., Martins, M. F., \& Vasconcelos, A. C. F. (2012). Entrevistas "em profundidade" na pesquisa qualitativa em administração: pistas teóricas e metodológicas. In: Simpósio de Administração da Produção, Logística e Operações Internacionais. FGV.

Ore, O., \& Sposato, M. (2021). Opportunities and risks of artificial intelligence in recruitment and selection. International Journal of Organizational Analysis.

Poisat, P., \& Mey, M. R. (2017). Electronic human resource management: Enhancing or entrancing? SA Journal of Human Resource Management, 15(1), 1-9.

Redondo, R. J. P. (2014). Una relación desequilibrada: la selección de personal en tiempos de crisis. methaodos. Revista de Ciencias Sociales, 2(1), 23-35.

Reis, B. F., \& Graminho, V. M. C. (2019). A inteligência artificial no recrutamento de trabalhadores: o caso Amazon analisado sob a ótica dos direitos fundamentais. Seminário Internacional, 1-21.

Robertson, I. T., \& Smith, M. (2001). Personnel selection. Journal of Occupational and Organizational Psychology, 74(4), 441-472.

Roth, P. L., Bobko, P., Van Iddekinge, C. H., \& Thatcher, J. B. (2016). Social media in employee-selection-related decisions: A research agenda for uncharted territory. Journal of Management, 42(1), 269-298.

Shackleton, V., \& Newell, S. (1994). European management selection methods: A comparison of five countries. International Journal of Selection and Assessment, 2(2), 91102.

Silva, A. H., \& Fossá, M. I. T. (2015). Análise de conteúdo: Exemplo de aplicação da técnica para análise de dados qualitativos.Qualit@s, 17(1), 1-14. 
Soete, B. D., Lievens, F., \& Druart, C. (2013). Strategies for dealing with the diversityvalidity dilemma in personnel selection: Where are we and where should we go? Revista de Psicología del Trabajo y de las Organizaciones, 29(1), 3-12.

Strohmeier, S., \& Piazza, F. (2015). Artificial intelligence techniques in human resource management-a conceptual exploration. In Intelligent Techniques in Engineering Management (pp. 149-172).

Torres-López, S. et al. (2014). Técnicas formales y de inteligencia artificial para la gestión de recursos humanos en proyectos informáticos. Revista Cubana de Ciencias Informáticas, 8(3), 41-52.

Triviños, A. N. S. (2008). Introdução à pesquisa em ciências sociais: a pesquisa qualitativa em educação. São Paulo: Atlas.

Valente, J. Inteligência artificial e o impacto nos empregos e profissões. Agência Brasil. https://agenciabrasil.ebc.com.br/geral/noticia/2020-08/inteligencia-artificial-e-o-impactonos-empregos-e-profissoes

Van Dyke, T., \& Strick, S. (1988). New concepts to old topics: Employee recruitment, selection and retention. Hospitality Education and Research Journal, 12(2), 347-360.

Weinstein, D. (2012). The psychology of behaviorally-focused résumés on applicant selection: Are your hiring managers really hiring the 'right' people for the 'right'jobs? Business Horizons, 55(1), 53-63.

Yin, R. K. (2015). Estudo de Caso: Planejamento e métodos. Porto Alegre: Bookman, 5.

Zanella, L. C. H. (2009). Metodologia de estudo e de pesquisa em administração. Florianópolis: Departamento de Ciências da Administração/UFSC. 